\title{
Editorial
}

\section{Gute Nachrichten}

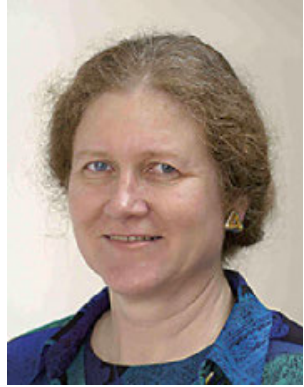

Bekanntlich ist die Therapie von Entzündungen und Schmerzen gerade bei den chronischen Erkrankungen nicht immer erfolgreich und zudem nebenwirkungsträchtig. Begründet wird dies durch die außerordentlich komplexen Entzündungsprozesse, die sich bei den verschiedenen Erkrankungen durchaus unterscheiden. Das aktuelle Heft gibt einige neue Anregungen zu Optionen aus der Phytotherapie und Einblicke in deren Mechanismen. Die Multitarget-Wirkungen von pflanzlichen Extrakten sind vermutlich gerade bei chronischen Prozessen von großem Vorteil, hier sollte verstärkt geforscht werden. Eine gute Nachricht in diesem Zusammenhang ist, dass ein bekanntes pflanzliches Kombinationspräparat mit den Anwendungsgebieten akute und subakute rheumatische Erkrankungen (z.B. Lumbago, Ischialgien, Neuralgien) wieder erhältlich ist.

Erfreulich ist auch die Entwicklung der Wahrnehmung der Phytotherapie in der medizinischen Fachpresse. So findet sich beispielsweise in einem aktuellen Heft der MMW mit dem Schwerpunkt Erkältung ein Übersichtsartikel zur leitliniengerechten Therapie von Schnupfen und Sinusitis [1]. Hier werden neben den chemisch definierten Arzneimitteln auch Phytopharmaka mit Myrtol, Cineol und
Pelargonium-sidoides-Extrakt sowie ein Fünffach-Extrakt aufgeführt und positiv bewertet. Die entsprechende europäische Leitlinie von 2007 wird erwähnt [2], und die neueren klinischen Studien werden korrekt zusammengefasst. In einem weiteren Artikel dieses Heftes werden Hamamelis-Extrakte und Flohsamenschalen im Zusammenhang mit proktologischen Erkrankungen empfohlen. Dieser konstruktive Umgang mit Phytopharmaka trägt dazu bei, dass das Interesse der Ärzte an der Phytotherapie wachgehalten wird und die Kenntnisse nicht verlorengehen.

Ich wünsche mir deshalb sehr, dass die Gesellschaft für Phytotherapie noch viele Informationen zur Phytotherapie in den verschiedenen medizinischen Leitlinien positionieren kann.

Karin Kraft

\section{Literatur}

1 Dippold N, Klimek L. Leitliniengerechte Therapie von Schnupfen und Sinusitis. MMW Fortschritte der Medizin 2017: 1: 37-40

2 Fokkens W, Lund V, Mullol J. EP3OS 2007: European position paper on rhinosinusitis and nasal polyps 2007. A summary for otorhinolaryngologists. Rhinology 2007: 45: 97-101 